\title{
Modeling Urban Road Risky Driving Behaviors in China with Multi-agent Microscopic Traffic Simulation
}

\author{
$\mathrm{Xia} \mathrm{Li}^{*}$, Ruibin $\mathrm{Bai}^{\dagger}$, Peer-Olaf Siebers ${ }^{\dagger}$ and Christian Wagner ${ }^{\dagger}$ \\ * International Doctoral Innovation Centre \\ University of Nottingham Ningbo China \\ Ningbo 315100, China \\ Email: XiaLi@nottingham.edu.cn
}

\begin{abstract}
Typical driving behaviors such as car-following and lane-changing can be described based on common concepts. But these behaviors could be different from driver to driver, from nation to nation due to different individual influencing factors (e.g. age, gender, driving age, mood) and situational influencing factors (e.g. weather, congestion, respect for law). Studies show that drivers who have higher level of "driving discourtesy" (i.e rudeness and aggressiveness) have higher probability of performing risky driving behaviors including traffic rule violations. In this paper, we propose a model named Driving Discourtesy Model (DDM). In this model, a new indicator is defined to measure the "driving discourtesy". With a probability distribution method, we are able to estimate the probability of performing risky driving behaviors of each vehicle based on a vehicle's individual influencing factors and situational factors. A multi-agent traffic simulation is developed to test DDM. The experiment results show that risky driving behaviors including speeding, lane-changing for taking speed advantages and driving on hard shoulder can be simulated effectively using DDM.
\end{abstract}

\section{INTRODUCTION}

Existing microscopic traffic simulation models have primarily been focusing on simulating time-space behaviors close to reality. Car-following behavior has been studied since early 1950s. Researchers had gained good understanding on this behavior via Physics point of view (e.g. eqution of motion). Psycho-spacing models (e.g. in [1], [2]) were proposed to help to describe car-following behavior via Psychophysics point of view. By introducing knowledge from Physics, Psychology, Cognitive Science and Social Science, simulation models are able to simulate traffic phenomena for more generic case. But driving behaviors could be different from driver to driver, from region to region and it is believed that time-space behaviors could be affected by many factors. Ranney [3] proposed a conceptual model that describes factors influencing the car-following behavior. These factors can be categorized into two types: individual factors and situational factors. Individual factors are unchanged constants over short period of time such as age, gender, driving skill, vehicle size etc. Situational factors are changed from time to time such as weather, road condition, hurry and distraction, impairment (alcohol, drugs, stress, fatigue, etc.). Ranney's conceptual model show some liner-like relations between those factors and car-following behaviors that individual factors become less important as the density increases while situational factors become more important but lose impacts when traffic becomes congested.

Feng et al. [4] investigate the psychometric properties of the Driving Anger Scale (DAS; [5]) and its relationship with aggressive driving in Chinese context with six subscales, which are discourtesy, traffic obstructions, hostile gestures, slow driving, police presence and illegal driving. Qu et al. [6] investigate correlation between individual factors (e.g. age, driving year) and possibility of engaging in risk driving. Zhang et al. [7] reported that drivers who have traffic incident history are more aggressive and less skillful than those without having incident during their driving experience. $\mathrm{Hu}$ et al. [8] perform experiment to investigate how positive and negative emotions and mood would influence driver's risky driving behavior through risk perception and risk attitude in China. Their results indicate that negative affect showed a stronger effect than positive affect; negative emotion raised driver's perception of traffic risk; and negative mood creates impact on attitude toward risky driving and self-reported risky driving behavior. Jie at el. [9] compares driving behaviors and saturation flow between drivers in China and Netherlands. They captured some differences between drivers in China and Netherlands at signalized intersections. They also suggested that when adopting microscopic traffic model in China, researchers should modify car-following behaviors. This includes using larger reaction time and lower free flow speed, performing more cautious following behaviors and possibly other values for preferred acceleration, deceleration and desired velocity. Also, the lane-changing model should represent a mixture of hesitating, aggressive and illegal lanechanging behaviors.

Not all drivers are rational and in normal manner all the time. Sometimes they would perform risky driving behaviors due to their individual status and situational factors. It is important to find out the connection between influencing factors and risky driving behaviors. Therefore, we propose a new model named "Driving Discourtesy Model" (DDM). With this model, we are able to simulate these risky driving behaviors and even predict them to some extent. This paper is organized as follows: Section II discusses the methodology of DDM. Section III discusses the modifications of an existing car-following model and lane-changing model using DDM. Section IV discusses the development of traffic simulation model. Section V discusses model validation and comparison of model with and without DDM. Section VI concludes our 
findings. Section VII discusses the future plan.

\section{METHODOLOGY}

In this paper, three types of driving behaviors are examined with DDM. They are: speeding (speed-limit violation), hardshoulder misuse and free lane-changing. The first two behaviors are typical risky behaviors that are commonly found in urban traffic in China. Free lane-changing behavior is not typically considered as a risky driving behavior but it has certain correlation with risky driving because in most cases drivers desire to take speed or position advantage through lane-changing. Drivers who do frequent free lane-changing are believed to have relatively less patience or are more aggressive than those who would rather follow the leading vehicles.

\section{A. Modeling Driving Behaviors using "Driving Discour-} tesy"

In order to build the "connection" between influencing factors and risky driving behaviors, we define a variable named $D D$ which denotes the "Driving Discourtesy" of a driver. High value of $D D$ means the driver has a higher probability to perform risky driving behaviors while low value refers to a lower probability. The value of $D D$ can be affected by many influencing factors including both individual and situational factors. Each factor may have different level of impact on increasing the probability of performing risky driving behaviors based on its weight. Thus $D D$ can be calculated via the following equation:

$$
D D_{x, t}=D D_{0}+\sum_{k=1}^{n}\left(w_{F_{k}} * S_{F_{k}}\right)
$$

where $D D_{x, t}$ denotes driving discourtesy value of vehicle $x$ at time $t, D D_{0}$ denotes the base value, $w_{F_{k}}$ and $S_{F_{k}}$ denote the weight and value of the $k$-th factor respectively. $D D_{0}$ represents the level of disrespect to traffic law. It ranges from 0 to 1 where 0 means low disrespect to traffic law and 1 refers high disrespect. In our study we assume that drivers in China generally have high level disrespect to the traffic law. So we set the default value of $D D_{0}$ to 1 .

\section{B. Selection of Influencing Factors}

Some factors have big impacts on engaging risky driving behaviors while some others are not. Some factors are essential for engaging risky driving behaviors in China while some are not. Therefore, we first select influencing factors that could be used and describe their relations to those risky behaviors based on some previous studies (e.g. [3-4], [1012]). The selected individual factors are listed in Table I and situational factors are listed in Table II. In these two tables, $P 1, P 2$ and $P 3$ denotes the probability of speeding, free lane-changing and hard-shoulder misuse respectively. $H, R H, L, R L$ and $U C$ denote "high", "relatively higher", "low", "relatively low" and "unclear" respectively. In traffic psychology, negative moods such as anger, stress and depression are studied separately. Here we simplify them into one factor named "negative mood" to make our modeling simpler.
TABLE I: Individual Factors and Their Relations to Driving Behaviors

\begin{tabular}{|l|l|l|l|l|}
\hline Factors & Condition & P1 & P2 & P3 \\
\hline \multirow{3}{*}{ Age } & $<30$ & H & H & UC \\
& $30 \leq$ Age $<45$ & RL & RL & UC \\
& $\geq 45$ & L & L & UC \\
\hline \multirow{2}{*}{ Gender } & Male & H & H & UC \\
& Female & L & L & UC \\
\hline \multirow{2}{*}{ Driving Age } & $<2$ years & L & L & L \\
& $\geq 2$ years & RH & UC & UC \\
\hline \multirow{2}{*}{ Incident History } & 0 & L & L & L \\
& $\geq 3$ & RH & RH & RH \\
\hline
\end{tabular}

TABLE II: Situational Factors and Their Relations to Driving Behaviors

\begin{tabular}{|l|l|l|l|l|}
\hline Factors & Condition & P1 & P2 & P3 \\
\hline \multirow{2}{*}{ Vehicle Type } & Truck & L & L & L \\
& Car & RH & RH & RH \\
\hline \multirow{2}{*}{ Urgency } & Yes & L & L & L \\
& No & H & H & H \\
\hline \multirow{2}{*}{ Negative Mood } & Low & L & L & L \\
& High & H & H & H \\
\hline \multirow{2}{*}{ Weather } & Bad & L & L & L \\
& Good & RH & RH & RH \\
\hline \multirow{2}{*}{ Time of Day } & Day & H & H & UC \\
& Night & RL & RL & RL \\
\hline \multirow{2}{*}{ Congestion } & Congested & L & L & L \\
& Uncongested & H & H & L \\
\hline
\end{tabular}

\section{Value Normalization}

Different factors take values from different domains. For example, factors such as age and driving age have different domains from that of driver's gender. One problem regarding this value representation is that a same value of different factors does not have equal impact on risky driving behaviors. Therefore it is necessary to do value normalization of these factors. The integer set $[1,2,3,4,5]$ is popular and widely used in survey to represent the level of importance of an item. Therefore we use the similar idea where 1 refers to the lowest level impact, 3 is the medium level and 5 is the highest level. The value normalization of the factors are described in Table. III.

\section{Weight Determination}

Each factor may have different level of impacts on increasing the probability of performing risky driving behaviors based on its "weight". Therefore, a weight should be determined for each factor. A proportional method [10] is adopted to do this. The proportional method is summarized in Table. IV. By using the proportional weight determination method, the weight of each factor can be obtained (Table. V).

\section{E. Calculation of Risky Driving Action Probability}

Now we are able to calculate $D D$ using Equation (1). Ranney 's conceptual model [3] shows some linear-like relations between the probability of driving behaviors and influencing factors. Based on this concept, we use a cumulative uniformed probability distribution function (known as $\mathrm{CDF}$ ) to establish the "connection" between $D D$ and the risky driving action probability. The CDF is indicated in Figure 1. 
TABLE III: Value Normalization of The Factors

\begin{tabular}{|c|c|c|}
\hline Factor & Value & Normalized Value \\
\hline \multirow{3}{*}{ Age } & $\in[20,30)$ & 5 \\
\hline & $\in[30,45)$ & 3 \\
\hline & $\in[45,60)$ & 1 \\
\hline \multirow{2}{*}{ Gender } & Male & 5 \\
\hline & Female & 1 \\
\hline \multirow{2}{*}{ Driving Age } & $\in[2,10)$ & 3 \\
\hline & $\in[0,2)$ & 1 \\
\hline \multirow{3}{*}{ Incident History } & $\in[3,5)$ & 5 \\
\hline & $\in[1,3)$ & 3 \\
\hline & $=0$ & 1 \\
\hline \multirow{2}{*}{ Vehicle Type } & Car & 3 \\
\hline & Truck & 1 \\
\hline \multirow{3}{*}{ Urgency } & Big & 5 \\
\hline & Small & 3 \\
\hline & None & 1 \\
\hline \multirow{3}{*}{ Anger/Stress } & High & 5 \\
\hline & Medium & 3 \\
\hline & Low & 1 \\
\hline \multirow[t]{2}{*}{ Time of Day } & $\in$ & 5 \\
\hline & $\begin{array}{l}{[\text { ram, } 9 \text { am }] \text { or }[5 \mathrm{pm}, 7 \mathrm{pm}]} \\
\text { other time }\end{array}$ & 3 \\
\hline \multirow{2}{*}{ Weather } & Good & 5 \\
\hline & Bad & 1 \\
\hline \multirow{3}{*}{ Congestion } & Free Flow & 5 \\
\hline & Dense But No Congestion & 3 \\
\hline & Congested & 1 \\
\hline
\end{tabular}

TABLE IV: Proportional Method for Weight Determination

\begin{tabular}{|l|l|l|}
\hline Factor A (\%) & Factor B (\%) & Definition \\
\hline $50 \%$ & $50 \%$ & A and B are equally important \\
\hline $60 \%$ & $40 \%$ & A is moderately more important than B \\
\hline $70 \%$ & $30 \%$ & A is strongly more important than B \\
\hline $80 \%$ & $20 \%$ & A is very strongly more important than B \\
\hline $90 \%$ & $10 \%$ & A is extremely more important than B \\
\hline $50+z \%$ & $50-z \%$ & intermediate terms $(0<z<50)$ \\
\hline
\end{tabular}

TABLE V: Weights of The Factors

\begin{tabular}{|l|l|l|l|}
\hline Factor & weights & Factor & weights \\
\hline Age & 0.0887 & Gender & 0.0860 \\
Driving Age & 0.0800 & Incident History & 0.1018 \\
Vehicle Type & 0.0824 & Urgency & 0.1264 \\
Negative Mood & 0.1218 & Time of Day & 0.0840 \\
Weather & 0.1038 & Congestion & 0.1251 \\
\hline
\end{tabular}

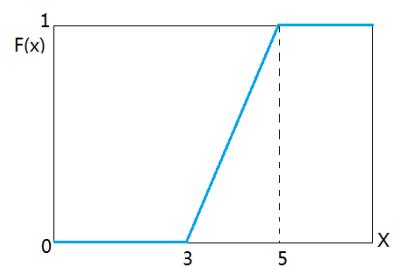

Fig. 1: Linear Relation Between $D D$ And Risky Driving Action Probability

The function in Figure 1 can be written by the following mathematical equation:

$$
F(D D)=\left\{\begin{aligned}
0 & \text { if } 0 \leq D D \leq 3 \\
\frac{D D-3}{5-3} & \text { if } D D \in(3,5) \\
1 & \text { if } D D=5
\end{aligned}\right.
$$

where $F(D D)$ is the function for the probability calculation.

\section{ModificAtion OF EXISTING MODEL}

\section{A. Modification of Car-Following Model}

The Intelligent Driving Model (IDM) [11] [12] is selected as the basic car-following model. In the original IDM, the acceleration of a vehicle at time $t+T$ can be described as follows:

$$
a_{i d m}(t+T)=a_{\text {free }}(t+T)-b(t+T)
$$

where $a_{\text {free }}(t+T)$ denotes the free road acceleration and $b(t+T)$ denotes the deceleration performed to avoid collision. $a_{\text {free }}(t+T)$ can be written as follows:

$$
a_{\text {free }}(t+T)=a_{\max }\left[1-\left(\frac{v}{v_{\text {desired }}}\right)^{\delta}\right]
$$

and $b(t+T)$ can be written as follows:

$$
b(t+T)=a_{\text {free }}\left[\frac{\left.s_{0}+v T_{\text {reaction }}+\frac{v \Delta v}{2 \sqrt{a_{\text {max }} b_{c o m}}}\right]}{s}\right]
$$

The descriptions of other parameters used in the model are indicated in Table VI.

\section{TABLE VI: Parameter Description of IDM}

\begin{tabular}{|l|l|}
\hline Parameter & Description \\
\hline$a_{\max }$ & maximum acceleration $\left(\mathrm{m} / \mathrm{s}^{2}\right)$ \\
$b_{\text {com }}$ & comfortable deceleration $\left(\mathrm{m} / \mathrm{sec}^{2}\right)$ \\
$v$ & current velocity at time $t+T(\mathrm{sec})$ \\
$v_{\text {desired }}$ & desired velocity $(\mathrm{m} / \mathrm{s})$ \\
$v_{l}$ & current velocity of its leading vehicle at time $t+T(\mathrm{~m} / \mathrm{s})$ \\
$s$ & current actual gap to its leading vehicle $(\mathrm{m})$ \\
$s_{0}$ & desired minimum gap space $(\mathrm{m})$ \\
$T_{\text {reaction }}$ & reaction time $(\mathrm{sec})$ \\
$\delta$ & acceleration exponent \\
\hline
\end{tabular}

Because higher value of $D D$ implies driver has higher probability to perform risky driving behaviors. We redefine a set of modification methods to recalculate those parameters above by incorporating DD factors (see Table VII).

TABLE VII: The Modifications of The Parameters In IDM

\begin{tabular}{|l|l|l|}
\hline Parameter & $\begin{array}{l}\text { IDM's Value Setting (order } \\
\text { by: normal, Timid, Aggressive, } \\
\text { Truck) }\end{array}$ & Mofication Method \\
\hline$a_{\max } / b_{\max }$ & $1.4,1.0,2.0,0.7$ & $\begin{array}{l}1.2 *\left(1+\frac{D D-3}{3}\right) \text { for small vehicles } \\
0.5 *\left(1+\frac{D D-3}{3}\right) \text { for large vehicles }\end{array}$ \\
\hline$b_{\text {com fort }}$ & $2.0,1.0,3.0,2.0$ & $2.0 *\left(1+\frac{D D-3}{3}\right)$ for all vehicles \\
\hline$s_{0}$ & $2.0,4.0,1.0,4.0$ & $3.0 *\left(1-\frac{D D-3}{3}\right)$ for small vehicles \\
& $1.0 *\left(1-\frac{D D-3}{3}\right)$ for large vehicles \\
\hline$T_{\text {reaction }}$ & $1.5,1.8,1.0,2.0$ & $2.0 *\left(1-\frac{D D-3}{3}\right)$ for small vehicles \\
& $120,100,140,85$ (highway) & $\begin{array}{l}\text { speedLimit } *\left(1+\frac{D D-3}{3}\right) \text { for small } \\
\text { vehicles } \\
\text { speedLimit } *\left(1+\frac{D D-3}{3}\right) * \beta \text { for large } \\
\text { vehicles, where } \beta=0.9\end{array}$ \\
\hline$v_{0}$ & &
\end{tabular}

\section{B. Modification of Lane-Changing Model}

The basic concept of the lane-changing model in this paper is based a widely accepted model proposed in [13]. Assume at time $t$, there is a gap on the target lane. If the subject vehicle plans to move into this gap, at time $t+\Delta T$, this lanechanging maneuver would create two new gaps: gap between the subject vehicle and its new leader, defined as $g_{l}$; and gap between the subject vehicle and its new follower, defined as 
$g_{f}$. The lane-changing is feasible if and only if $g_{l}(t+\Delta T) \geq$ $g_{l, \text { min }}$ and $g_{f}(t+\Delta T) \geq g_{f, \text { min }}$ where $g_{l, \text { min }}$ and $g_{f, \text { min }}$ denote minimal acceptable gap spacing respectively. If the gap is feasible, acceleration is calculated via the following equation:

$$
a_{\text {need }}=\left(d_{x}+v_{m p}+a_{m p} / 2-v_{s}\right) * 2
$$

where $d_{x}$ is the distance to the position of merge-point (i.e position where vehicle moves into a feasible gap for lanechanging), $v_{m p}$ and $a_{m p}$ denote speed and acceleration of the subject vehicle at merge-point respectively, $v_{s}$ is the velocity of the subject vehicle. If $a_{\text {need }} \leq a_{\max }$ (equivalent to $a_{\max }$ in IDM), the vehicle moves into this gap to complete lane-changing. "Lane-Change Plan" is used in the original model to enable the "communication" amongst participated vehicles (i.e. the subject vehicle, potential new follower and new leader). This is done by creating a distinguished object and keep itself updated from time to time. Plan will be removed when it becomes invalid (i.e. participated vehicle no longer exists). During simulation, the number of plans can be very large especially when vehicles are waiting for lane-changing at merging area. In our simulation, "visible distance" is used so that vehicle "scans" to find all gaps within its "visible distance" when it plans to change lane. Therefore no "Lane Change Plan" is needed. The shaded area in Figure 2 indicates the "visible area" of a vehicle. Another modification is when $D D>3$, there exits probability of using hard shoulder of road when traffic is getting dense. Also, driver who has higher value of $D D$ is more likely to adopt more aggressive acceleration when moving into a gap for lane-changing and accept smaller gap space when a new leader is moving into the lane.

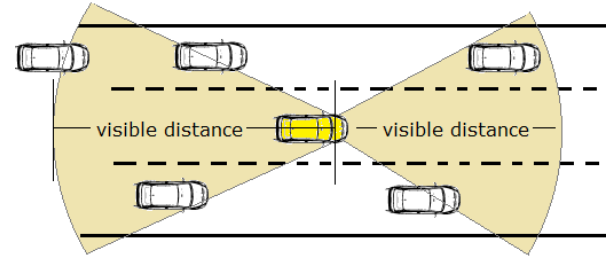

Fig. 2: Distance-Based Gap Seeking

\section{Simulation}

Multi-agent modeling is used to develop our microscopic traffic simulation. Each agent represents a "mixed object" of a vehicle and its driver. Each agent is distinguished in terms of their properties(e.g. vehicle length, age, gender, mood) and behaviors(e.g. velocity adoption strategy, probability of performing risky driving behaviors). To make our model stochastic rather than a deterministic, randomization techniques are used for the initialization of agents and lanechanging behaviors when selecting target lane if more than one lane is feasible. The simulation is implemented using Anylogic(version 6), which is a multi-method simulation modeling software.
A 2880-meter road segment (i.e. in our simulation, one pixel equals to 1 meter in real world) is designed which is very similar to a local bridge called Zhaobaoshan Bridge in Ningbo, China. The road structure is indicated in Figure 3. The gray part is the hard-shoulder area. Two traffic signal lights are set at both west and east sides of the bridge with cycling time of $30 \mathrm{sec}$ to ease our simulation in stead of using different settings (15 $\mathrm{sec}$ and $1 \mathrm{~min}$ ) in reality.

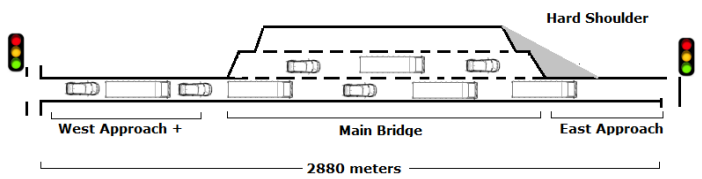

Fig. 3: Road Structure

A set of parameters are defined in the simulation. These parameters can be categorized into three types: system con$\operatorname{trol}(\mathrm{S})$, characteristics $(\mathrm{C})$ and vehicle control. Some essential parameters and their initialization methods are indicated in Table VIII (see vehicle control parameters in Table VI).

TABLE VIII: Essential Parameters And Initialization Methods

\begin{tabular}{|c|c|c|}
\hline Type & Parameter & Initialization Method \\
\hline \multirow{6}{*}{$\mathrm{S}$} & Speed Limit & $\begin{array}{l}60 \mathrm{~km} / \mathrm{h} \text { by default (i.e. } 16.7 \mathrm{pixel} / \mathrm{sec} \\
\text { in simulation) }\end{array}$ \\
\hline & Vehicle Arrival Rate & uniform probability distribution \\
\hline & Incident & disabled by default \\
\hline & Agents Update time Interval & $1 \mathrm{sec}$ \\
\hline & Level of Congestion & 1 (not congested) \\
\hline & Stop Zone & length of 40 meters by default \\
\hline \multirow{8}{*}{$\mathrm{C}$} & Age & $\begin{array}{l}\text { triangular probability distribution, where } \\
\min =20, \max =50 \text { and } \text { mode }=40\end{array}$ \\
\hline & Gender & probability of male is 0.7 by default \\
\hline & Driving Age & $\begin{array}{l}\text { triangular probability distribution, where } \\
\min =2, \max =10 \text { and } \text { mode }=5\end{array}$ \\
\hline & Incident History & $\begin{array}{l}\text { triangular probability distribution, where } \\
\min =0, \max =5 \text { and } \text { mode }=3\end{array}$ \\
\hline & Small/Large Vehicle Ratio & six days obtained from field study \\
\hline & Length & $\begin{array}{l}5 \text { meters for small vehicle and } 10 \text { meters } \\
\text { for large vehicle }\end{array}$ \\
\hline & Urgency & $\begin{array}{l}\text { probability of no urgency is } 80 \% \text { and } \\
19.5 \% \text { in a small hurry based on speeding } \\
\text { behavior analysis of field study data }\end{array}$ \\
\hline & Negative Mood & $\begin{array}{l}A S=F_{i}+F_{s} \text { where } F_{i} \text { denotes internal } \\
\text { factors and } F_{s} \text { denotes situational factors. } \\
F_{s}=0 \text { by default. }\end{array}$ \\
\hline
\end{tabular}

"Stop Zone" is defined in our simulation. If a vehicle enters the "Stop Zone" meanwhile the traffic signal light is switching to "red", whether a vehicle slows down to stop or accelerate to pass through end line of the lane depends on the "driving discourtesy" of the driver: If $D D<3$, the vehicle slows down to stop; otherwise the vehicle accelerates to pass the end line. An observation field is set up in our simulation to measure the characteristics of the traffic flow (i.e. mean velocity, flow rate, density).

\section{EXPERIMENT AND RESUlts}

\section{A. Validation}

An experiment is conducted to validate the traffic simulation model. The simulation is running for four times with four different arrival rates $(0.3,0.5,0.7,0.9)$. The simulation 
is running for 6 days where two 1-hour time slots are selected each day (one in the morning and one in the afternoon). Flow rate and density data are collected every 5 minutes. We first validate the model against traffic theory. The results are indicated in Figure 4. The simulation is valid in terms of representing the fundamental relations between speed, density and flow when DDM is enabled. The simulation is also valid when DDM is disabled.

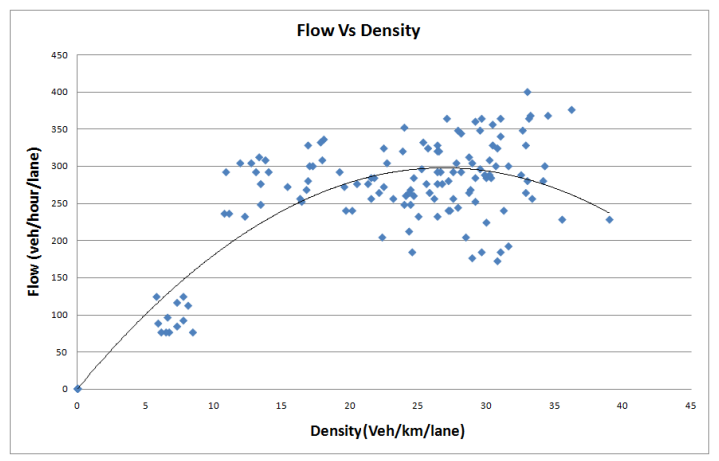

(a) DDM Enabled

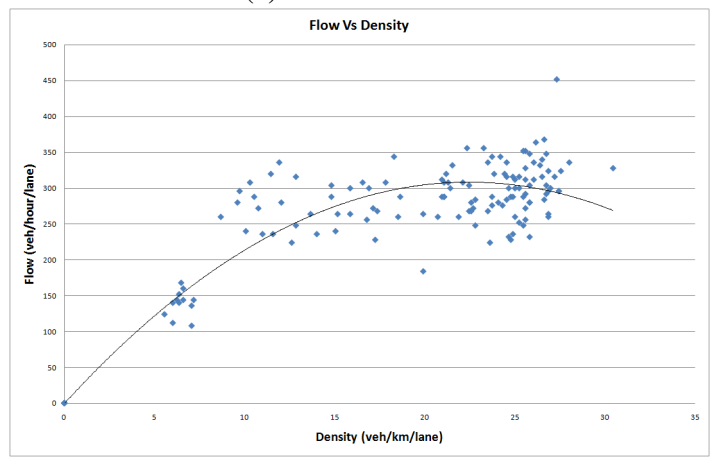

(b) DDM Disabled

Fig. 4: Flow Rate Vs Density

\section{B. Comparison Experiment}

Four scenarios are defined for the formal experiment: DDM enabled with an incident, DDM disabled with an incident, DDM enabled without incident and DDM disabled without incident. Speeding count uses a per-timecount method. The results in Figure 5a) indicate that more speeding behaviors are captured with the increase of the arrival rate. With same arrival rate, more speeding behaviors are captured when an incident is simulated. No speeding behaviors are captured when DDM is disabled. Free lanechanging refers to lane-changing for taking speed or position advantage. The results in Figure 5b) show that more free lane-changing are captured with the increase of the arrival rate. With same arrival rate, more free lane-changing are captured when DDM is enabled. More free lane-changing are captured when an incident is simulated. When DDM is enabled, more hard shoulder misuse are captured with the increase of the arrival rate except for when the arrival rate is 0.7 . More hard shoulder misuses are captured when an incident is simulated. When DDM is disabled, no hard shoulder misuse with the arrival rate of $0.3,0.5$ and 0.7 . However, a few of vehicles are captured to misuse the hard shoulder area when the arrival rate is 0.9 .

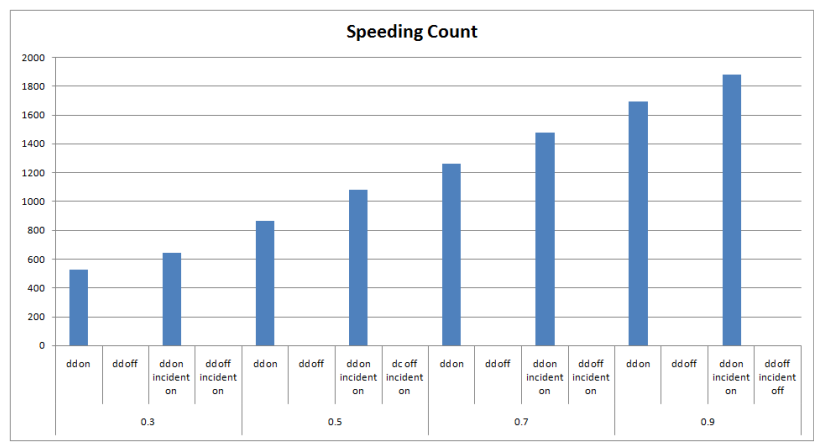

(a) Speeding Counts Histogram (per vehicle count)

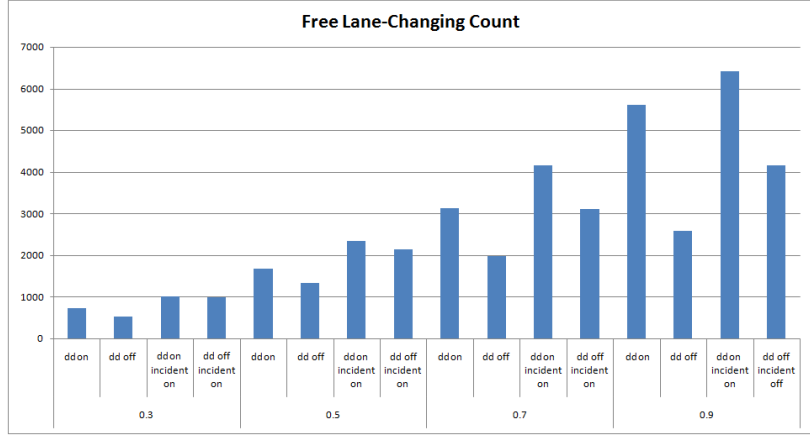

(b) Free Lane-Changing Counts Histogram (per lane-changing count)

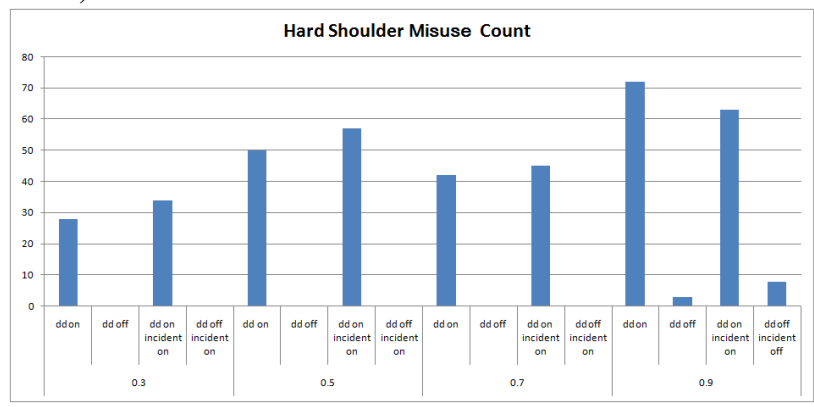

(c) Hard Shoulder Misuse Counts Histogram (per vehicle count)

Fig. 5: Histogram of Risky Driving Behaviors

Imperative lane-changing is triggered when the current lane is blocked ahead or the current lane is not allowed to take turning. The results in Figure 6 show that a lot more imperative lane-changing are captured when an incident is simulated but it becomes less when DDM is disabled. Figure 7 shows the result of the mean travel time. The results indicate that when the arrival rate is 0.3 , the difference on mean travel time is not significant regardless whether DDM or incident is on or not. When the arrival rate increases to $0.5,0.7$ and 0.9 , the mean travel time is longer when DDM is enabled.

\section{CONCLUSION}

From the simulation results we could see that when DDM is disabled no speeding and hard shoulder misuse are 


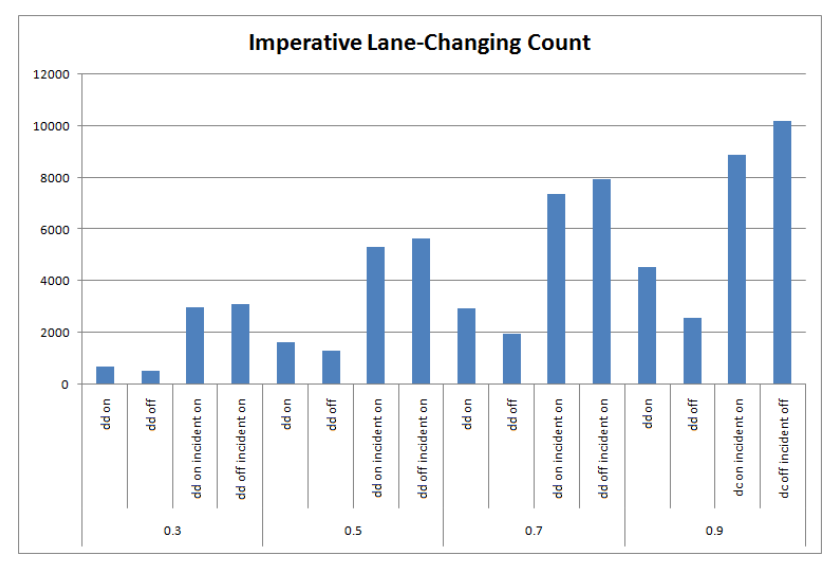

Fig. 6: Imperative Lane-Changing Counts Histogram (per time count)

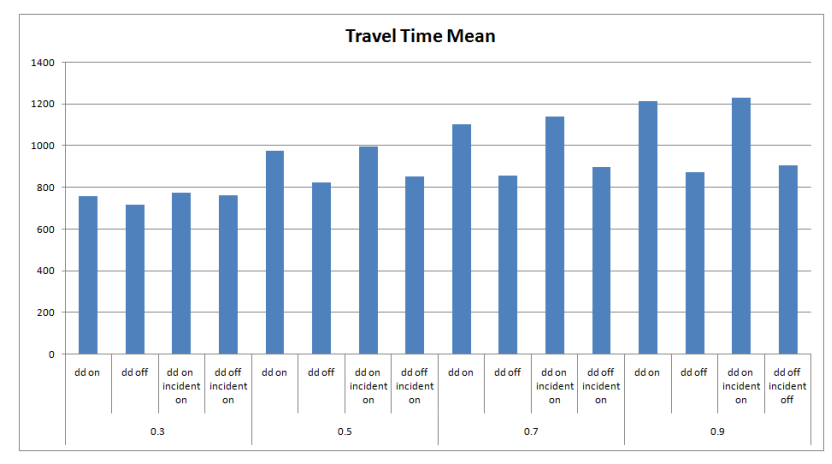

Fig. 7: Travel Time Mean Histogram

captured with arrival rates of $0.3,0.5$ and 0.7 . This is because drivers of all vehicles are modeled as rational persons tend not to perform any risky driving. A few cases for hard shoulder misuse are seen during the experiment when the arrival rate is 0.9 . This is because, a waiting queue is formed at the merging area and some drivers unintentionally enter the hard shoulder area by just following their leader who select the left most lane after lane-changing by mistake. Incident along with lower arrival rates may not cause more risky driving behaviors because the incident does not block the road completely and there is adequate time and space to digest the downstream traffic flow before the road gets congested. When DDM is enabled a little congestion is captured at merging area due to more free lane-changing behaviors and we are able to identify drop on capacity. If congestion lasts for longer, the value of $D D$ would increase due to the increase of negative mood. With DDM, we are able to build connections between the influencing factors and risky driving behaviors.

\section{FUTURE RESEARCH}

Different behaviors may have different influencing factors and different weights. The selection of factors and determination of weights should be verified more carefully by surveying more drivers and experts. Multi-factor model would be introduced in the next version model, so that different behaviors are treated differently with different combination of factors and different value of weights. A crisp set is used to represent normalized values of those factors. This may not be the best choice for value normalization. Fuzzy set would be a better choice when describe the level of impact on risky driving (i.e high, medium, low). In this study, we assume that the connection between $D D$ and the probability of risky driving behaviors is linear based on Ranney's conceptual model in [3]. We should further examine this linear correlation assumption with more literature reviews and field studies.

\section{ACKNOWLEDGMENT}

This work was carried out at the International Doctoral Innovation Centre (IDIC). The author acknowledges the financial support from Ningbo Education Bureau, Ningbo Science and Technology Bureau, China's MOST and The University of Nottingham. The work is also partially supported by EPSRC grant no EP/L015463/1 and Ningbo Science \& Technology Bureau grant no 2012B10055 .

\section{REFERENCES}

[1] S. Jin, D.-H. Wang, Z.-Y. Huang, and P.-F. Tao, "Visual angle model for car-following theory," Physica A: Statistical Mechanics and its Applications, vol. 390, no. 11, pp. 1931 - 1940, 2011.

[2] R. G. Hoogendoorn, S. P. Hoogendoorn, K. A. Brookhuis, and W. Daamen, "Longitudinal driving behavior under adverse conditions: A close look at psycho-spacing models," Procedia - Social and Behavioral Sciences, vol. 20, no. 0, pp. 536 - 546, 2011.

[3] T. A. Ranney, "Psychological factors that influence car-following and car-following model development," Transportation Research Part F: Traffic Psychology and Behaviour, vol. 2, no. 4, pp. 213 - 219, 1999.

[4] F. Li, X. Yao, L. Jiang, and Y. Li, "Driving anger in china: Psychometric properties of the driving anger scale (das) and its relationship with aggressive driving," Personality and Individual Differences, vol. 68 , no. 0 , pp. $130-135,2014$.

[5] J. L. Deffenbacher, E. R. GETTING, and R. S. Lynch, "Development of a driving anger scale," Psychological reports, vol. 74, no. 1, pp. 83-91, 1994

[6] W. Qu, Y. Ge, C. Jiang, F. Du, and K. Zhang, "The dula dangerous driving index in china: An investigation of reliability and validity," Accident Analysis \& Prevention, vol. 64, no. 0, pp. 62 - 68, 2014.

[7] L. Zhang, J. Wang, F. Yang, and K. Li, "A quantification method of driver characteristics based on driver behavior questionnaire," in Intelligent Vehicles Symposium, 2009 IEEE, June 2009, pp. 616-620.

[8] T.-Y. Hu, X. Xie, and J. Li, "Negative or positive? the effect of emotion and mood on risky driving," Transportation Research Part F: Traffic Psychology and Behaviour, vol. 16, no. 0, pp. 29 - 40, 2013.

[9] L. Jie, H. van Zuylen, Y. Chen, and R. Lu, "Comparison of driver behaviour and saturation flow in china and the netherlands," Intelligent Transport Systems, IET, vol. 6, no. 3, pp. 318-327, September 2012.

[10] A. Aldian and M. A. Taylor, "A consistent method to determine flexible criteria weights for multicriteria transport project evaluation in developing countries," Journal of the Eastern Asia Society for Transportation Studies, vol. 6, pp. 3948-3963, 2005.

[11] M. Treiber, A. Hennecke, and D. Helbing, "Microscopic simulation of congested traffic," in Traffic and granular flow99. Springer Berlin Heidelberg, 2000, pp. 365-376.

[12] A. Kesting, M. Treiber, and D. Helbing, "Agents for traffic simulation," arXiv preprint arXiv:0805.0300, 2008.

[13] P. Hidas, "Modelling vehicle interactions in microscopic simulation of merging and weaving," Transportation Research Part C: Emerging Technologies, vol. 13, no. 1, pp. 37 - 62, 2005. 